\title{
Micro-electromechanical Determination of the Possible Remanent Strain and Polarization States in Polycrystalline Ferroelectrics and the Implications for Phenomenological Constitutive Theories
}

\author{
CHAD M. LANDIS, * JiANXIN WANG AND JiANSHUN SHENG \\ Department of Mechanical Engineering and Materials Science, MS 321, Rice University, P.O. Box 1892, Houston, TX 77251, USA
}

\begin{abstract}
A micro-electromechanical constitutive model for polycrystalline ferroelectric ceramics is implemented to determine the remanent strain and remanent polarization states that are possible in a ferroelectric polycrystal. The underlying crystal structure of the single crystals is assumed to be tetragonal, but the methods developed in this paper can be applied to other crystal structures as well. The full multi-axial set of remanent strain saturation states possible in an untextured polycrystal is determined. It is argued and demonstrated that the remanent polarization saturation level is dependent on the prevailing remanent strain state. The possible uniaxial combinations of remanent strain and polarization are determined from the micro-electromechanical model. The results of these investigations are then applied within a phenomenological constitutive framework and the constitutive response under coupled electromechanical loading is predicted.
\end{abstract}

Key Words: ferroelectrics, micro-electromechanics, constitutive behavior, strain saturation, polarization saturation

\section{INTRODUCTION}

$\mathbf{T}$ HE need for accurate yet simple constitutive laws for ferroelectric ceramics arises in a number of problems that require the resolution of the inhomogeneous electrical and mechanical fields in ferroelectric materials. Such problems range from the prediction of poling patterns around electrodes in ferroelectric actuator designs to the determination of switching zones near crack tips that govern failure in these materials. Due to the relative complexity of the constitutive response of ferroelectrics, these types of problems will most likely be solved by implementing the finite element method or some other numerical technique. Hence, it is desirable to keep the mathematical description of the constitutive response as simple as possible while maintaining the prominent physical features of the material behavior.

Along these lines, a phenomenological constitutive framework for the coupled multi-axial constitutive response of polycrystalline ferroelectrics has been developed by Landis (2002). As with any phenomenological constitutive framework, this theory sets up constraints that enforce the laws of thermodynamics and contains undetermined functions that can be tailored

*Author to whom correspondence should be addressed E-mail: landis@rice.edu to predict specific material behavior. The theory is sufficiently general as to admit a wide range of material response, and it is up to the practitioner to provide the appropriate inputs required to model a specific material.

One of the fundamental features of ferroelectric constitutive response is that the range of remanent strain and remanent polarization states that are achievable by the material is limited. Take for example, the simple uniaxial application of electric field to an unpoled polycrystalline sample of ferroelectric material. Initially, at small electric fields, the material responds as a linear dielectric with the electric displacement proportional to the applied electric field. As the electric field reaches the coercive field of the material, domain switching commences and the electric displacement and remanent polarization increase rapidly with very small increases in electric field. However, unlike plastic deformation in metals, the remanent polarization is limited to a finite value. This finite level of remanent polarization is referred to as polarization saturation or lock-up. As the remanent polarization nears saturation, the electric field begins to increase more dramatically and eventually linear dielectric response about the remanent polarization state is approached again. For purely mechanical loading, similar response between the applied stress and the resulting strain occurs as well. Finally, for coupled electrical and mechanical loading, 
the situation is more complicated with the saturation level of remanent polarization dependent on the remanent strain state.

It is well established that the saturation behavior of ferroelectric ceramics arises due to the underlying crystal structure of the material, see for example Kamlah (2001). Furthermore, since a phenomenological constitutive framework cannot predict the remanent saturation states of the material a priori, the saturation conditions must be determined by some other means and entered into the theory. Without such restrictions, phenomenological constitutive models will allow unrealistic combinations of remanent strain and polarization to arise. Since the experimental determination of the saturation states in ferroelectrics would be difficult if not impossible, micro-electromechanical investigations of the possible remanent states in these materials are the most appropriate procedure to determine the saturation conditions.

A number of micro-electromechanical methods exist that model the behavior of ferroelectric polycrystals as the averaged response of randomly oriented single crystal grains. In all cases, the constitutive response of the single crystals is intended to model the domain switching mechanism that gives rise to remanent strain and polarization in these materials. Then, the polycrystalline averages range in sophistication from Taylor and Reuss-like models where interaction between crystals is neglected (Hwang et al., 1995; Lu et al., 1999), to self-consistent methods where grain to grain constraints are accounted for in an average sense (Hwang et al., 1998; Huber et al., 1999), to finite element simulations of many cubic single crystals where interactions are fully accounted for (Hwang and McMeeking, 1998, 1999). In principle, any of these micro-electromechanical models can be applied to the task of determining the complete range of remanent strain and remanent polarization states that are attainable by a polycrystalline ferroelectric ceramic.

The remainder of this paper is organized as follows. Section 2 will review the salient features of the microelectromechanical constitutive model of Huber et al. (1999) that will be applied to determine the polycrystalline saturation states. Section 3 will present the methodology used to ascertain the possible remanent states and the associated results that are obtained from the computations. Section 4 will review the phenomenological constitutive framework of Landis (2002) and demonstrate how the micro-electromechanical results can be implemented within the theory. Predictions of electromechanical constitutive response will be made in this section as well. Lastly, Section 5 will offer a discussion of the results and implications of the model for different crystal structures and textured polycrystals.

\section{THE MICRO-ELECTROMECHANICAL MODEL}

Throughout this work, subscripted indices will be used to represent the Cartesian components of vectors and tensors. Summation over repeated subscripted indices is assumed. Superscripts will be used to enumerate quantities like variant volume concentrations or switching transformation systems. Summation over repeated superscripted indices should not be assumed and will be symbolized explicitly.

The model presented here for the constitutive response of ferroelectric polycrystals has been developed in detail by Huber et al. (1999). This constitutive law consists of two primary components, a constitutive law for single crystals and a self-consistent averaging technique to determine the polycrystalline response. The single crystal constitutive law accounts for domain switching between the crystallographic variants of the crystal in a manner analogous to continuum slip models for plasticity in metal single crystals. Then, the selfconsistent averaging model proposed by Hill (1965, 1967) and implemented by Hutchinson (1970) for metal polycrystals is applied to determine the constitutive behavior of the polycrystal.

\subsection{Single Crystal Constitutive Law}

Throughout this paper it is assumed that the underlying lattice structure of the single crystal is tetragonal. This assumption will of course limit the applicability of the results to tetragonal materials, however the models can be readily generalized to other crystal structures as well. In this work, we are only concerned with the possible remanent states of the material and not the electromechanical loading required to generate them. Therefore, conceptual and computational simplicity within the single crystal constitutive model will be afforded if the linear elastic, piezoelectric and dielectric response of a single variant is taken to be isotropic. This implies that within the model, the piezoelectric coefficients $d_{k i j}$ all go to zero for a single domain single crystal. Of course, this is not the actual physical situation. The assumption of isotropy of the linear properties of the single crystals renders the results generated in this work to be only approximate. However, even for the case of anisotropic single crystals, we assume that the saturation states of the polycrystal are most prominently dependent on the underlying geometry and spatial orientations of the unit cells. Hence, the results for the polycrystalline remanent saturation states generated for the simplified case of single crystals with isotropic linear properties are assumed to be a reasonable approximation to the actual saturation states for polycrystals assembled from grains with anisotropic piezoelectric linear properties. 
The single crystal constitutive law is analogous to continuum slip plasticity models with the added effects of polarization and strain saturation. In order to derive the single crystal constitutive law, it is assumed that the stress and electric field are uniform and both the total and remanent parts of the strain and electric displacement are the volume averages over the entire crystal. Note that by assuming the stress and electric field within the single crystal are uniform, the electromechanical interactions between domains in the crystal are neglected. As illustrated in Figure 1, six tetragonal variants can exist in any single crystal. Hence, the remanent strain and polarization of the crystal are

$$
\varepsilon_{i j}^{r, s c}=\sum_{\mathrm{I}=1}^{6} c^{I} \varepsilon_{i j}^{r(I)} \text { and } P_{i}^{r, s c}=\sum_{\mathrm{I}=1}^{6} c^{I} P_{i}^{r(I)}
$$

where $c^{I}$ represents the volume concentration of the Ith variant. Here, the superscript $s c$ is used to denote single crystal values in order to differentiate between the polycrystalline values of the analogous quantities introduced in the next section. Also, the remanent strains and polarizations of each of the variants are given by

$$
\begin{aligned}
\varepsilon_{i j}^{r(1)} & =\varepsilon_{i j}^{r(2)}=\varepsilon_{0}\left(3 \delta_{i 1} \delta_{j 1}-\delta_{i j}\right) / 2, \\
\varepsilon_{i j}^{r(3)} & =\varepsilon_{i j}^{r(4)}=\varepsilon_{0}\left(3 \delta_{i 2} \delta_{j 2}-\delta_{i j}\right) / 2, \quad \text { and } \\
\varepsilon_{i j}^{r(5)} & =\varepsilon_{i j}^{r(6)}=\varepsilon_{0}\left(3 \delta_{i 3} \delta_{j 3}-\delta_{i j}\right) / 2
\end{aligned}
$$

and

$$
\begin{aligned}
& P_{i}^{r(1)}=P_{0} \delta_{i 1}, \quad P_{i}^{r(2)}=-P_{0} \delta_{i 1}, \quad P_{i}^{r(3)}=P_{0} \delta_{i 2}, \\
& P_{i}^{r(4)}=-P_{0} \delta_{i 2}, \quad P_{i}^{r(5)}=P_{0} \delta_{i 3} \quad \text { and } \quad P_{i}^{r(6)}=-P_{0} \delta_{i 3}
\end{aligned}
$$

where the 1,2 and 3 coordinate directions are parallel to the $\langle 100\rangle$ crystal directions as drawn in Figure $1, \delta_{i j}$ is the Kronecker delta, $\varepsilon_{0}$ is the spontaneous strain of a single variant and can be given in terms of the lattice parameters as $\varepsilon_{0}=2(c-a) /(c+2 a)$, and $P_{0}$ is the spontaneous polarization of a single variant. With these definitions of the remanent strains and polarizations for the individual variants, the remanent strain and polarization of the entire crystal will be zero if there are equal volume concentrations of each of the variants. Finally, under the assumption that the variants are elastically and electrically isotropic (nonpiezoelectric), the total strain and electric displacement of the single crystal are

$$
\varepsilon_{i j}^{s c}=\frac{1}{2 \mu} \sigma_{i j}^{s c}-\frac{v}{2 \mu(1+v)} \sigma_{k k}^{s c} \delta_{i j}+\varepsilon_{i j}^{r, s c}
$$

and

$$
D_{i}^{s c}=\kappa E_{i}^{s c}+P_{i}^{r, s c}
$$

where $\mu, v$ and $\kappa$ are the isotropic shear modulus, Poisson's ratio and dielectric permittivity respectively. Note that Equations (2.4) and (2.5) differ from those

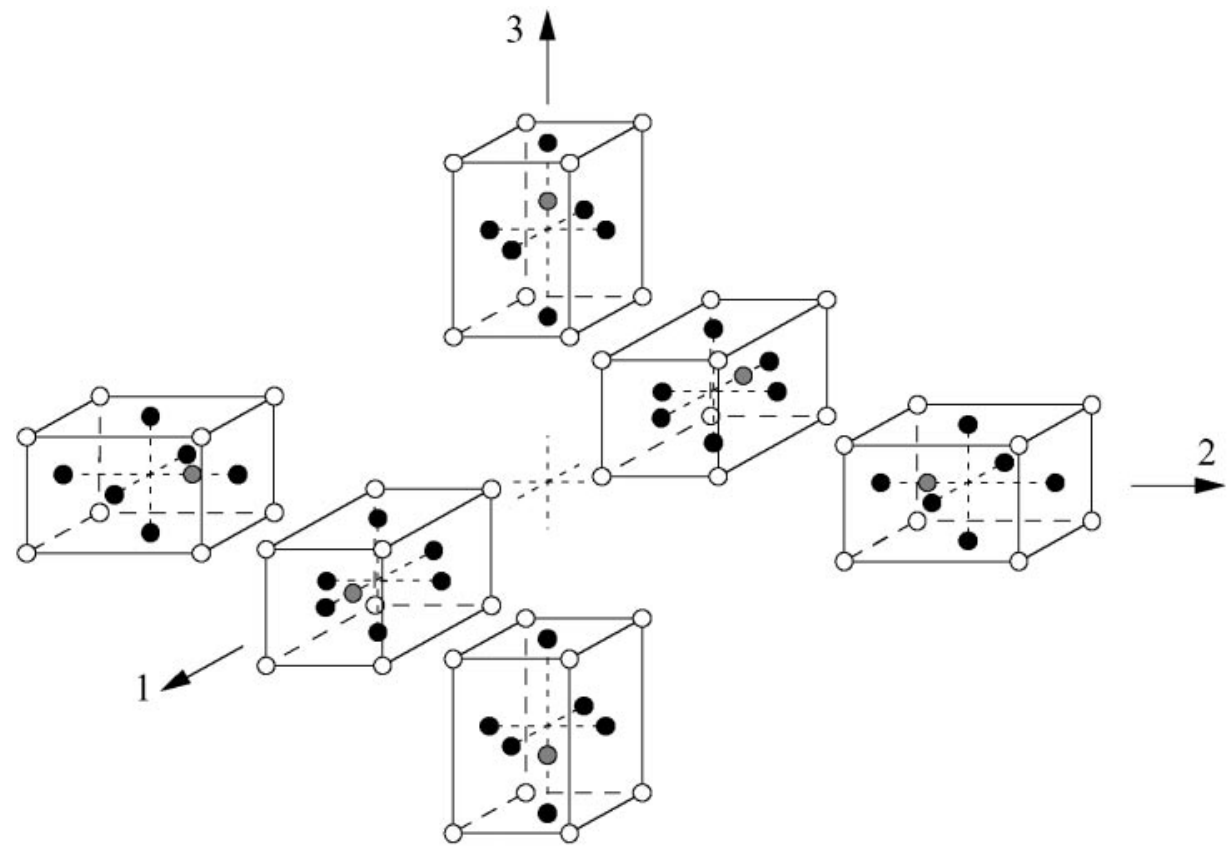

Figure 1. The six possible tetragonal variants in a single crystal. Here the 1,2 and 3 coordinate directions correspond to the $\langle 100\rangle$ crystal directions. 
appearing in Huber et al. (1999) due to the simplification that the linear properties of the variants are isotropic.

In order to determine how the volume concentrations of the variants can change with applied loading, we will first consider the free energy of the crystal. Under isothermal conditions, the Helmholtz free energy of the single crystal, $\Psi^{s c}$, is equal to the stored electromechanical energy of the crystal and is given as

$$
\begin{aligned}
& \Psi^{s c}\left(\varepsilon^{s c}, \varepsilon^{r, s c}, D^{s c}, P^{r, s c}\right) \\
& \quad=\mu\left(\varepsilon_{i j}^{s c}-\varepsilon_{i j}^{r, s c}\right)\left(\varepsilon_{i j}^{s c}-\varepsilon_{i j}^{r, s c}\right) \\
& \quad+\frac{\mu \nu}{1-2 \nu}\left(\varepsilon_{k k}^{s c}\right)^{2}+\frac{1}{2 \kappa}\left(D_{i}^{s c}-P_{i}^{r, s c}\right)\left(D_{i}^{s c}-P_{i}^{r, s c}\right)
\end{aligned}
$$

Note that isotropy of the linear properties and the deviatoric nature of the remanent strains $\left(\varepsilon_{k k}^{r, s c}=0\right)$ have been used to derive Equation (2.6). The dissipation rate in the single crystal is given as the work rate due to applied stresses and electric fields minus the free energy rate. By applying the previously stated assumptions, the dissipation rate can be shown to be

$$
\begin{aligned}
\dot{w}^{D} & =\sigma_{i j}^{s c} \dot{\varepsilon}_{i j}^{s c}+E_{i}^{s c} \dot{D}_{i}^{s c}-\dot{\Psi}^{s c}=\sigma_{i j}^{s c} \dot{\varepsilon}_{i j}^{r, s c}+E_{i}^{s c} \dot{P}_{i}^{r, s c} \\
& =\sum_{\mathrm{I}=1}^{6} \dot{c}^{\mathrm{I}}\left(\sigma_{i j}^{s c} \varepsilon_{i j}^{r(\mathrm{I})}+E_{i}^{s c} P_{i}^{r(\mathrm{I})}\right)
\end{aligned}
$$

Note that thirty different switching transformations are possible, i.e. each of the six variants can switch to the other five. Furthermore, if a transformation is occurring, then the volume concentrations of the variants being switched to and from increase and decrease respectively at exactly the same rates. By applying these facts, it is then possible to rewrite the dissipation rate and define the transformation driving forces, $G^{\alpha}$, as

$$
\begin{aligned}
\dot{w}^{D} & =\sum_{\alpha=1}^{30} \dot{f}^{\alpha}\left(\sigma_{i j}^{s c} \Delta \varepsilon_{i j}^{r(\alpha)}+E_{i}^{s c} \Delta P_{i}^{r(\alpha)}\right) \\
& =\sum_{\alpha=1}^{30} \dot{f}^{\alpha} G^{\alpha} \quad \text { where } \quad G^{\alpha} \equiv \sigma_{i j}^{s c} \Delta \varepsilon_{i j}^{r(\alpha)}+E_{i}^{s c} \Delta P_{i}^{r(\alpha)}
\end{aligned}
$$

Here, $\alpha$ numbers the thirty possible transformation systems and the $\Delta$ represents the difference in the following quantity between the variant being transformed to and that being transformed from. For example, take $\alpha=1$ to represent the transformation from variant 1 to variant 3 . Then, $\Delta \varepsilon_{i j}^{r(\alpha=1)}=3 \varepsilon_{0}\left(\delta_{i 2} \delta_{j 2}-\delta_{i 1} \delta_{j 1}\right) / 2$ (note that this is a pure shear strain $), \Delta P_{i}^{r(\alpha=1)}=P_{0}\left(\delta_{i 2}-\delta_{i 1}\right)$ and the volume concentration rates are $\dot{c}^{(I=1)}=-\dot{f}^{(\alpha=1)}$ and $\dot{c}^{(\mathrm{I}=3)}=\dot{f}^{(\alpha=1)}$. Note that six of these transformation systems account for $180^{\circ}$ switching, and the other twenty-four model $90^{\circ}$ switching. Furthermore, due to the assumption of isotropic linear properties, the driving forces identified in Equation (2.8) do not contain terms associated with changes in the linear properties as in Huber et al. (1999), and are identical to the driving forces for switching employed in Hwang et al. (1995) and $\mathrm{Lu}$ et al. (1999).

It is assumed that if a quantity of a given variant exists, i.e. $c^{I}>0$, then it is possible to incrementally switch that variant into one of the other five. Furthermore, this switching between variants is assumed to occur only if such a transformation results in a characteristic rate of non-negative dissipation. Specifically, a transformation system is potentially active if

$$
G^{\alpha}=G_{c}^{\alpha} \rightarrow f^{\alpha} \geq 0
$$

and the transformation system is inactive if

$$
G^{\alpha}<G_{c}^{\alpha} \rightarrow f^{\alpha}=0
$$

If the volume concentration of a given variant vanishes, then the transformation systems that reduce the quantity of that variant cannot be activated. This feature enables the model to account for strain and polarization saturation at the single crystal level. Furthermore, driving forces greater than the critical levels are not permissible except under saturation conditions. In the absence of hardening of the transformation systems, i.e. when the $G_{c}^{\alpha}$ remain fixed during switching, the single crystal constitutive law requires the following inputs; the isotropic linear properties of a single tetragonal variant $\mu, v$ and $\kappa$, the critical driving forces to induce $180^{\circ}$ and $90^{\circ}$ transformation $G_{c}^{180}$ and $G_{c}^{90}$, the lattice parameters of a tetragonal variant that determine $\varepsilon_{0}$, and the spontaneous polarization of a single variant $P_{0}$. Ultimately, the possible saturation states of the material will only depend on $\varepsilon_{0}$ and $P_{0}$.

From Equations (2.1)-(2.10) it is a mathematical exercise to determine the transformation rates, $\dot{f}^{\alpha}$, in terms of the applied strain and electric displacement rates, and then forms for the single crystal tangent moduli follow. This procedure will not be given here, but it is presented clearly in Huber et al. (1999).

\subsection{The Self-consistent Polycrystal}

Using the single crystal constitutive law described above, a self-consistent model is applied to compute the overall electromechanical behavior for a polycrystal. For conceptual simplicity, the polycrystal is viewed as an infinite collection of randomly oriented single crystals, each individually subjected to homogenous states of stress, electric field, strain and electric displacement. The Cartesian components of the macroscopic field increments of the polycrystal are taken to be 
the volume averages of the Cartesian components of the corresponding increments in the single crystals. Each individual single crystal region is modeled as a spherical inclusion embedded in an infinite effective medium matrix. The tangent moduli of the effective medium are taken to be those of the polycrystal. Since neighboring grains are not modeled explicitly, the constraint interactions between grains are not determined directly in this model. Instead, each grain is constrained by the effective medium matrix, and in this sense the model accounts for grain-to-grain constraints in an averaged sense. Ultimately, the electromechanical state in each single grain will depend on the applied loading history and the orientation of the crystal. For more details of both the single crystal constitutive law and the self-consistent averaging method the reader is referred to Huber et al. (1999). The most significant feature of the self-consistent model for the polycrystal is that, given the current state of the material, it provides incremental constitutive relations of the form

$$
\dot{\sigma}_{i j}=c_{i j k l}^{t} \dot{\varepsilon}_{k l}-h_{k i j}^{t} \dot{D}_{k}
$$

and

$$
\dot{E}_{i}=-h_{i k l}^{t} \dot{\varepsilon}_{k l}+\beta_{i j}^{t} \dot{D}_{j}
$$

where $c_{i j k l}^{t}, h_{k i j}^{t}$ and $\beta_{i j}^{t}$ are the tangent electromechanical moduli of the polycrystal and $\dot{\sigma}_{i j}, \dot{E}_{i}, \dot{\varepsilon}_{i j}$ and $\dot{D}_{i}$ are the components of the increments of the stress, electric field, strain and electric displacement of the polycrystal. Note that no superscript is used to denote polycrystalline quantities.

\section{DETERMINATION OF POSSIBLE REMANENT STATES}

Since phenomenological constitutive laws do not predict any one specific material behavior a priori, it is up to the user to supply the appropriate information and constraints required to model a given material. For ferroelectric ceramics, the underlying crystal structure imposes geometric constraints on the states of remanent strain and remanent polarization that are achievable in the material. For example, consider a single crystal that is initially unpoled with equal volume concentrations of the six tetragonal variants. Under the application of a large tensile stress along a $\langle 100\rangle$ direction the ultimate remanent strain will be $2 \varepsilon_{0}$ along the tensile axis. However, for compressive loading, the remanent strain will only be $-\varepsilon_{0}$. Hence, for a $\langle 100\rangle$ loaded single crystal there is an asymmetry in the possible remanent strain states achievable in tension and compression of $2: 1$. Next, consider the possible remanent polarization states for the same single crystal under these two extreme remanent strain conditions. For the tensile remanent strain state described above, any remanent polarization state aligned with the tensile axis between $-P_{0}$ and $P_{0}$ is possible. In contrast, the remanent polarization aligned with the maximum compressive remanent strain axis must be zero. From this perspective, the remanent strain state sets boundaries within which the remanent polarization can exist. The question that we would like to address in this paper is; what are the full multi-axial remanent strain and remanent polarization states that are possible in a ferroelectric polycrystal?

Consider the two-dimensional schematic of a ferroelectric polycrystal illustrated in Figure 2. The arrows represent the spontaneous polarization directions of domain variants. Note that for tetragonal materials, the $c$-axes of the unit cells of the variants are aligned with this arrow. Furthermore, the spontaneous strains for two variants with opposite spontaneous polarization directions are identical. Upon cooling from above the Curie temperature every spontaneous polarization direction is equally likely to occur, this is represented by state A. In this reference state, the remanent strain and remanent polarization of the polycrystal are both equal to zero. If only $180^{\circ}$ switching is allowed to occur, then state $B$ can be achieved where the remanent strain remains at zero but the remanent polarization can increase. If $90^{\circ}$ switching is allowed to occur and a tensile stress is applied to state A, then the remanent strain can reach a maximum tensile value while the remanent polarization remains at zero as drawn in state C. Then, if an electric field is applied, $180^{\circ}$ switching can cause the polycrystalline remanent polarization to reach its maximum attainable level illustrated in state D. State E depicts the variant orientations for a sample that has been transformed from state A due to an applied compressive stress. Note again that the application of stress and no electric field to the reference state leaves the polycrystal with no net remanent polarization. Finally, state $\mathrm{F}$ illustrates the maximum polarization that can occur in the fully compressed material. It will be shown in the following subsections that the magnitudes of the maximum tensile remanent strain, maximum compressive remanent strain and maximum remanent polarization of the polycrystal are $\varepsilon_{t}=0.55 \varepsilon_{0}, \varepsilon_{c}=0.40 \varepsilon_{0}$ and $P_{\max }=0.83 P_{0}$. The relative magnitudes of the remanent polarization of the polycrystal in states $\mathrm{B}$ and $\mathrm{F}$ are also taken from the selfconsistent computations to be presented in this section.

Note that for all of the self-consistent computations presented in this paper, the initial volume concentrations of each variant type in every single crystal orientation are equal to one sixth. Hence, this circumstance defines the initial remanent strain and remanent polarization state of the material. Due to the isotropy of the single crystals, the remanent strain and polarization 


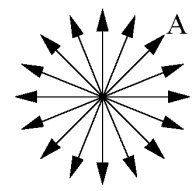

Reference state, as cooled from above the Curie temperature, $\varepsilon^{r}=0$ and $P^{r}=0$.

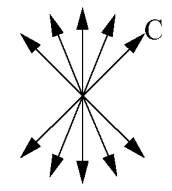

$\varepsilon^{r}=\varepsilon_{t}$ and

$P^{r}=0$

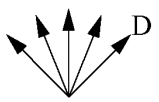

$\varepsilon^{r}=\varepsilon_{t}$ and
$P^{r}=P_{\max }$

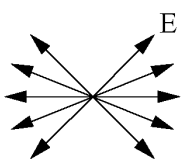

$\varepsilon^{r}=-\varepsilon_{c}$ and
$P^{r}=0$

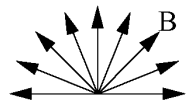

$\varepsilon^{r}=0$ and

$P^{r} \approx 2 P_{\max } / 3$

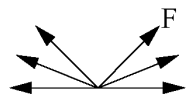

$\varepsilon^{r}=-\varepsilon_{c}$ and

$P^{r} \approx P_{\max } / 4$

Figure 2. A two-dimensional schematic of the single variant orientation distribution in a ferroelectric polycrystal and the effects on the remanent strain and remanent polarization states. The arrows represent the direction of the spontaneous polarization and the c-axis of the unit cell. Note that the spontaneous strains of oppositely directed variants are identical. Also notice that smaller numbers of arrows imply that the directions shown in a schematic contain a larger volume concentration. For example, the volume concentrations of the 5 arrows drawn in state $D$ and of the 16 drawn in state $A$ each sum to unity. Hence, the "weight" of the arrows in state $D$ is greater than those in state A.

of the polycrystal are simply the volume average of the remanent strain and polarization of the single crystals. Furthermore, the linear elastic and dielectric properties of the polycrystal are simply those of the single crystals as well. In order to probe for the possible remanent states of the polycrystalline material, we would like to be able to control the increments of the remanent strain and polarization. However, the self-consistent formulation requires either the increments of total strain and electric displacement or stress and electric field. Given the increments of remanent strain and remanent polarization that are desired, the following equations can be manipulated to determine the increments of total strain and electric displacement.

$$
\begin{gathered}
\dot{\sigma}_{i j}=2 \mu\left(\dot{\varepsilon}_{i j}-\dot{\varepsilon}_{i j}^{r}+\frac{v}{1-2 v} \dot{\varepsilon}_{k k} \delta_{i j}\right)=c_{i j k l}^{t} \dot{\varepsilon}_{k l}-h_{k i j}^{t} \dot{D}_{k} \\
\dot{E}_{i}=\frac{1}{\kappa}\left(\dot{D}_{i}-\dot{P}_{i}^{r}\right)=-h_{i k l}^{t} \dot{\varepsilon}_{k l}+\beta_{i j}^{t} \dot{D}_{j}
\end{gathered}
$$

Equations (3.1) and (3.2) represent nine equations for the nine unknown polycrystalline strain and electric displacement increments, $\dot{\varepsilon}_{i j}$ and $\dot{D}_{i}$, given the specified increments of the remanent quantities, $\dot{\varepsilon}_{i j}^{r}$ and $\dot{P}_{i}^{r}$, the applied volume strain, $\dot{\varepsilon}_{k k}$, and the tangent moduli supplied by the self-consistent averaging model, $c_{i j k l}^{t}, h_{k i j}^{t}$ and $\beta_{i j}^{t}$. Through the solution of these equations, any remanent strain and polarization path up to saturation can be traversed within the self-consistent model. In the following, it will be shown how this procedure is applied to find the full multi-axial set of remanent strain saturation states, and the uniaxial set of remanent polarization saturation states.

\subsection{Possible Multi-axial Remanent Strain States}

The goal of this first investigation is to map out the saturation conditions for remanent strain states between axisymmetric contraction, through pure shear remanent strain, and on to axisymmetric extension. To do this the following remanent strain invariants are introduced

$$
J_{2}^{e}=\left(\frac{2}{3} e_{i j}^{r} e_{i j}^{r}\right)^{1 / 2} \text { and } J_{3}^{e}=\left(\frac{4}{3} e_{i j}^{r} e_{j k}^{r} e_{k i}^{r}\right)^{1 / 3}
$$

Here $e_{i j}^{r}$ is the remanent strain deviator, $e_{i j}^{r}=\varepsilon_{i j}^{r}-$ $\delta_{i j} \varepsilon_{k k}^{r} / 3$. Note that $J_{2}^{e}$ is the effective remanent strain. With the definition of these two invariants, a full range of remanent strain saturation states can be probed by allowing the ratio of $J_{3}^{e} / J_{2}^{e}$ to vary from -1 (axisymmetric contraction) to 0 (pure shear) to 1 (axisymmetric extension). If we consider any volume conserving remanent strain in a coordinate system aligned with the principal remanent strain directions, then the remanent strain tensor and $J_{3}^{e} / J_{2}^{e}$ can be written as

$$
\begin{aligned}
& \varepsilon_{i j}^{r}=\left(\begin{array}{ccc}
\varepsilon^{r} & 0 & 0 \\
0 & b \varepsilon^{r} & 0 \\
0 & 0 & -(1+b) \varepsilon^{r}
\end{array}\right) \text { and } \\
& \frac{J_{3}^{e}}{J_{2}^{e}}=\frac{-\sqrt{3}\left(b+b^{2}\right)^{1 / 3}}{4^{1 / 6} \sqrt{1+b+b^{2}}} \operatorname{sgn}\left(\varepsilon^{r}\right)
\end{aligned}
$$

where $b$ and $\varepsilon^{r}$ can take on arbitrary values. The ratio $J_{3}^{e} / J_{2}^{e}$ only depends on $b$ and the sign of $\varepsilon^{r}$, and the limiting or saturation level of $\varepsilon^{r}$ will depend on 
$b$. Ultimately, every multi-axial volume conserving remanent strain state can be described by its effective ratio will always lie in the range $-1 \leq J_{3}^{e} / J_{2}^{e} \leq 1$.

Figures 3(a) and (b) plot results from self-consistent computations with the remanent strain invariant ratio $J_{3}^{e} / J_{2}^{e}$ held fixed throughout the entire simulation. Figure 3(a) illustrates the effective stress versus effective remanent strain response that occurs during such a simulation. During such purely ferroelastic response, only $90^{\circ}$ switching can take place and the critical driving force for $90^{\circ}$ switching is $G_{c}^{90}=3 \tau_{0} \varepsilon_{0}$, where $\tau_{0}$ is the critical resolved shear stress required to induce $90^{\circ}$ switching and $\varepsilon_{0}$ is the spontaneous strain of a single variant as defined in Section 2. At low levels of applied stress, the material responds in a linear elastic fashion and the remanent strain remains at zero. As the stress is increased, domain switching commences in favorably oriented single crystals at an effective stress level of approximately 2 to 2.2 times $\tau_{0}$. Switching proceeds at this stress level with some modest overall hardening until some of the single crystals reach strain saturation. At this point, the polycrystalline effective stress must remanent strain and eventually the remanent strain can no longer increase at all. This limiting level of remanent strain is the saturation level for the polycrystal and, as illustrated in Figure 3(a), is a function of the ratio $J_{3}^{e} / J_{2}^{e}$. Finally, Figure 3(b) plots the saturation level of the effective remanent strain versus the remanent strain invariant ratio $J_{3}^{e} / J_{2}^{e}$. Note that the asymmetry in the magnitude of remanent strain attainable in tension versus compression for the polycrystal is approximately $1.37: 1$.

(a)

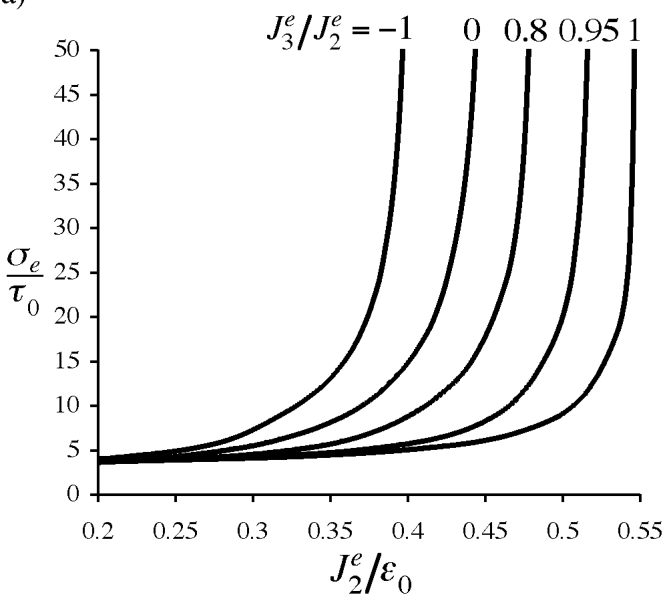
magnitude $J_{2}^{e}$ and by the ratio of $J_{2}^{e}$ and $J_{3}^{e}$, and this increase dramatically in order to increase the overall

\subsection{Possible Uniaxial Remanent Polarization States}

Given that Figure 3(b) illustrates the full multi-axial range of remanent strain states that are possible in a polycrystalline ferroelectric material with underlying tetragonal crystal structure, we now turn to the determination of the possible remanent polarization states. It is noted that the saturation level of the remanent polarization is dependent on the remanent strain state. For example, consider only axisymmetric states of remanent strain and remanent polarization aligned along the same axis. If the remanent strain is tensile, then the magnitude of the remanent polarization that is possible is maximized. However, if the remanent strain is compressive, then there is not "enough room" for the full remanent polarization to fit into this remanent strain state. In general, the polarization saturation conditions will be a function of the remanent strain invariant ratio $J_{3}^{e} / J_{2}^{e}$, the magnitude of the effective remanent strain $J_{2}^{e}$, and the direction of the remanent polarization with respect to the principal remanent strain directions. For a Cartesian coordinate system aligned with the principal directions of the remanent strain state, this remanent polarization direction can be specified by two independent components of the remanent polarization direction $n_{i}$. Hence, the normalized polarization saturation conditions can be written as $P_{\text {sat }} / P_{0}=\tilde{P}\left(J_{3}^{e} / J_{2}^{e}, J_{2}^{e} / \varepsilon_{0}, n_{1}, n_{2}\right)$. To fully investigate this four-dimensional parameter space is an important undertaking, but has not been carried out here. In this work, only uniaxial simulations have been performed.

To quantitatively determine the uniaxial remanent polarization saturation conditions, the following

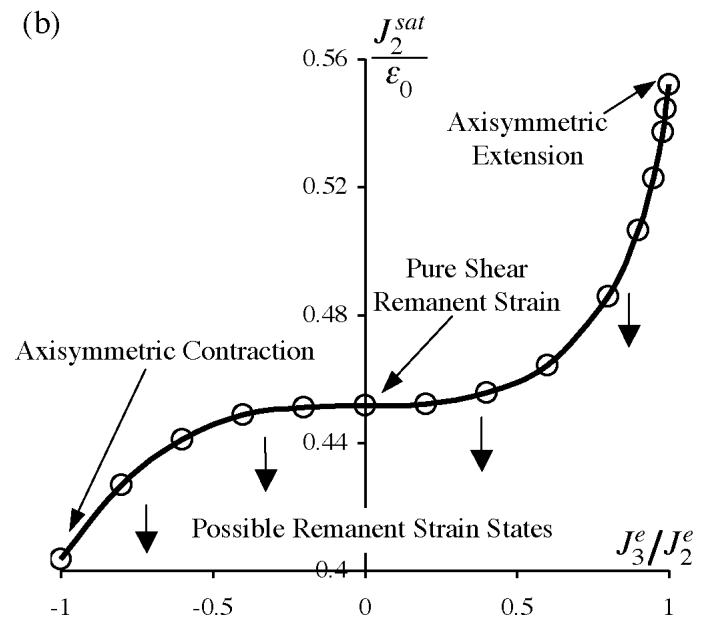

Figure 3. (a) The effective stress vs. the $J_{2}^{e}$ remanent strain invariant for a range of the ratio $J_{3}^{e} / J_{2}^{e}$. Pure axisymmetric contraction corresponds to $J_{3}^{e} / J_{2}^{e}=-1$, pure shear remanent strain is at $J_{3}^{e} / J_{2}^{e}=0$, and axisymmetric extension is $J_{3}^{e} / J_{2}^{e}=1$. The saturation levels of $J_{2}^{e}$ correspond to the asymptotic values as the effective stress goes to infinity. The critical shear stress $\tau_{0}$ is the shear stress when $90^{\circ}$ switching occurs in a single crystal such that $G_{c}^{90}=3 \tau_{0} \varepsilon_{0}$; (b) The asymptotic or saturation levels of $J_{2}^{e}$ as a function of $J_{3}^{e} / J_{2}^{e}$. Remanent strain states below the curve are possible in the material, but those states above the curve are unattainable. 
computations were performed. First, the self-consistent model material is loaded by either a uniaxial tensile or compressive stress in order to achieve an axisymmetric remanent strain state, i.e. the uniaxial remanent strain falls in the range $-0.40 \leq \varepsilon^{r} / \varepsilon_{0} \leq 0.55$ with $J_{3}^{e} / J_{2}^{e}=$ $\operatorname{sgn}\left(\varepsilon^{r}\right)$ and $J_{2}^{e}=\left|\varepsilon^{r}\right|$. Such uniaxial loadings correspond to the two extreme limits of $J_{3}^{e} / J_{2}^{e}$ as shown in Figure 3(b). Next, electric field and mechanical stress are applied in the appropriate proportions by manipulating Equations (3.1) and (3.2) such that the remanent strain state remains constant while the remanent polarization state is increased to saturation. Again, all loading and changes in remanency occur along the same axis. Hence, the polarization saturation conditions in such uniaxial loading situations are reduced to a one-dimensional parameter space, i.e. $P_{\text {sat }} / P_{0}=\tilde{P}_{\text {uni }}\left(\varepsilon^{r} / \varepsilon_{0}\right)=\tilde{P}\left(J_{3}^{e} / J_{2}^{e}=\operatorname{sgn}\left(\varepsilon^{r}\right), J_{2}^{e} / \varepsilon_{0}=\left|\varepsilon^{r}\right| / \varepsilon_{0}\right.$, $\left.n_{1}=0, n_{2}=0\right)$ where the loading axis is aligned with the $x_{3}$-direction.

Figure 4 plots the saturation levels of polarization $P_{\text {sat }}$ versus the magnitude of the uniaxial remanent strain $\varepsilon^{r}$. Note that the remanent polarization can be in one of the two opposing directions such that $-P_{\text {sat }} \leq P^{r} \leq P_{\text {sat }}$. The curve plotted on Figure 4 is exactly the function $\tilde{P}_{\text {uni }}\left(\varepsilon^{r} / \varepsilon_{0}\right)$ described previously. It is worth noting that a few points appearing on Figures $3(\mathrm{~b})$ and 4 are in agreement with results reported from other microelectromechanical computations. First, the magnitudes of the saturation levels of remanent strain in tension and compression quoted in Kamlah (2001) and Frölich (2001) are $\varepsilon_{t}=0.5520 \varepsilon_{0}$ and $\varepsilon_{c}=0.4035 \varepsilon_{0}$. Also, the maximum tensile remanent strain and the maximum remanent polarization for a polycrystal from $\mathrm{Lu}$ et al. (1999) are $\varepsilon_{t}=0.5514 \varepsilon_{0}$ and $P_{\max }=0.831 P_{0}$. The results presented here suggest that $\varepsilon_{t}=0.55 \varepsilon_{0}, \varepsilon_{c}=$ $0.40 \varepsilon_{0}$ and $P_{\max }=0.83 P_{0}$. Hence, the results presented here are in excellent agreement with other microelectromechanical models. This is to be expected when comparing models that apply the Reuss approximation (Frölich (2001) and Lu et al. (1999)) to models that assume homogeneous linear properties from grain to grain as in this work. Under these types of assumptions the saturation states of the material will only depend on the geometry of the underlying crystal structure and not on the specific details of the micro-model. Hence, any of these other micro-electromechanical models could be used to generate the results presented in Figures 3(b) and 4. Note that an actual ferroelectric polycrystal has inhomogeneous linear properties from grain to grain and hence the saturation conditions will depend on both the geometry of the crystal structure and ratios of the linear material properties that describe the anisotropy of the single crystal constituents. Hence the results presented in Figures 3(b) and 4 serve as reasonable approximations to the actual saturation conditions. Finally, one last result presented in Figure 4 demonstrates that the maximum amount of remanent polarization that can be achieved when the uniaxial remanent strain is at its maximum compressive level is approximately one quarter of $P_{\max }$, see Figure 2F. Specifically, $P_{\text {sat }}\left(\varepsilon^{r}=-0.40 \varepsilon_{0}\right)=0.21 P_{0}$.

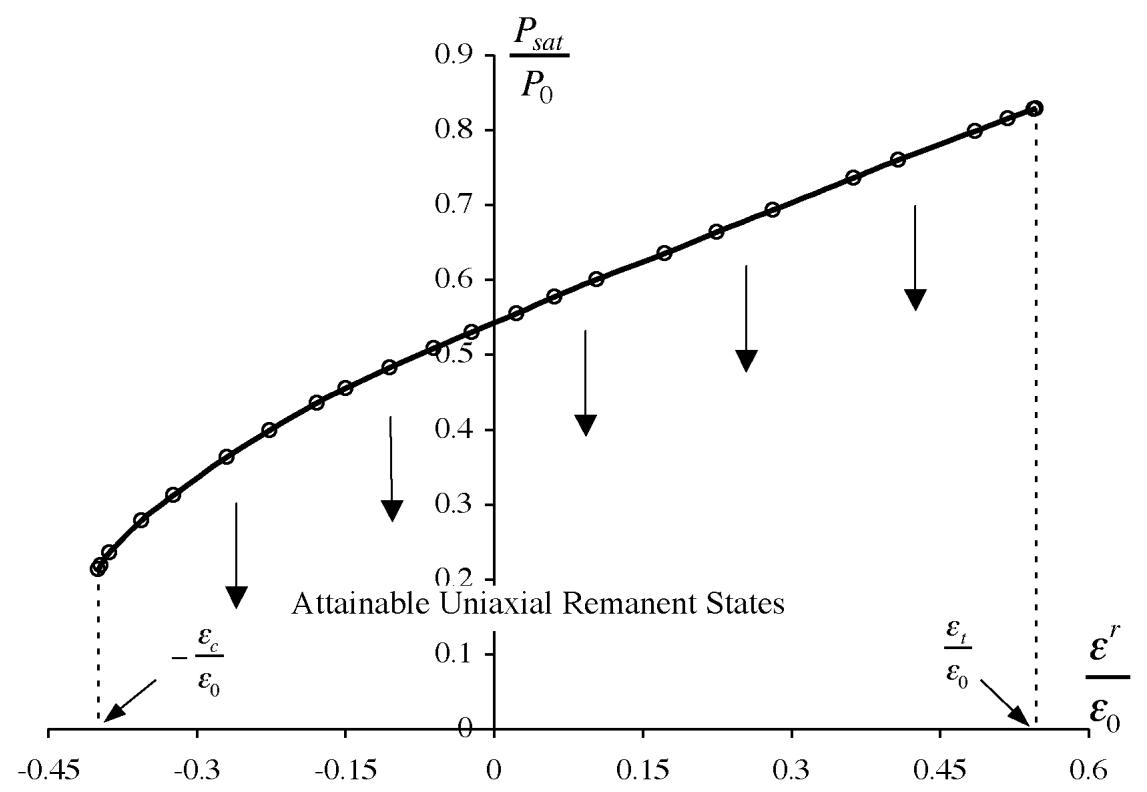

Figure 4. The saturation curve for states of remanent polarization as a function of remanent strain. Note that the uniaxial remanent strain ranges from $-0.40 \varepsilon_{0} \leq \varepsilon^{r} \leq 0.55 \varepsilon_{0}$ corresponding to the saturation states for $J_{3}^{e} / J_{2}^{e}=-1$ and $J_{3}^{e} / J_{2}^{e}=1$. This saturation curve is for remanent polarization states aligned along the remanent strain axis. Furthermore, $P_{\text {sat }}$ represents the magnitude of the maximum remanent polarization such that the remanent polarization aligned with the strain axis can take on values $-P_{\text {sat }} \leq P^{r} \leq P_{\text {sat }}$. 


\section{IMPLICATIONS FOR PHENOMENOLOGICAL THEORIES}

The goal of any phenomenological internal variable constitutive theory is to provide a relatively simple framework within which the laws of thermodynamics are satisfied and a wide range of material behaviors can be represented. The micro-electromechanical model described in the previous section cannot be considered simple because the number of internal variables, namely the volume concentrations of each variant type in each single crystal grain, is too large. The phenomenological framework developed in Cocks and McMeeking (1999) and Landis (2002) postulates that the minimum number of internal variables required to model fully coupled ferroelectric material behavior is nine (eight if the remanent strain is volume conserving), specifically the Cartesian components of the remanent strain and remanent polarization. The most general form of the framework will not be presented here and the reader is referred to Landis (2002) for all of the details. Instead, only the specific form of the theory used to model the PLZT material investigated by Lynch (1996) will be given here.

First, it is assumed that the Helmholtz free energy of the polycrystal is given as

$$
\begin{aligned}
\Psi= & \frac{1}{2} c_{i j k l}^{D}\left(\varepsilon_{i j}-\varepsilon_{i j}^{r}\right)\left(\varepsilon_{k l}-\varepsilon_{k l}^{r}\right)-h_{k i j}\left(D_{k}-P_{k}^{r}\right)\left(\varepsilon_{i j}-\varepsilon_{i j}^{r}\right) \\
& +\frac{1}{2} \beta_{i j}^{\varepsilon}\left(D_{i}-P_{i}^{r}\right)\left(D_{j}-P_{j}^{r}\right)+\Psi^{r}\left(\varepsilon_{i j}^{r}, P_{i}^{r}\right)
\end{aligned}
$$

where the linear elastic, piezoelectric and dielectric properties of the polycrystal, $c_{i j k l}^{D}, h_{k i j}$ and $\beta_{i j}^{\varepsilon}$ can each depend on the remanent state of the material. Note here that the isotropy of the linear material properties is no longer being assumed. The theory is greatly simplified if the following forms are taken for the inverse properties, $s_{i j k l}^{E}, d_{k i j}$ and $\kappa_{i j}^{\sigma}$

$$
\begin{gathered}
s_{i j k l}^{E}=\frac{1}{4 \mu}\left(\delta_{i k} \delta_{j l}+\delta_{i l} \delta_{j k}\right)-\frac{v}{2 \mu(1+v)} \delta_{i j} \delta_{k l} \\
\kappa_{i j}^{\sigma}=\kappa \delta_{i j} \\
d_{k i j}=\frac{d_{33}}{4} \frac{P^{r}}{P_{\max }}\left(3 n_{i} \delta_{j k}+3 n_{j} \delta_{i k}-2 n_{k} \delta_{i j}\right)
\end{gathered}
$$

where

$$
P^{r}=\sqrt{P_{i}^{r} P_{i}^{r}} \quad \text { and } \quad n_{i}=P_{i}^{r} / P^{r}
$$

Here, $d_{33}$ is the piezoelectric coefficient when the material reaches its maximum remanent polarization of $P_{\max }=0.83 P_{0}$. Note that this form for the piezoelectric coefficients has $d_{31}=-d_{33} / 2$ and $d_{15}=3 d_{33} / 2$. This is a reasonable assumption based on measured values in polycrystals, and this assumption can be relaxed at the added expense of complexity within the theory. Note that the "remanent potential" $\Psi^{r}$ only depends on the remanent state of the material and is intended to account for the free energy associated with the changes in stored energy due to intergranular constraints that generate internal residual stresses and electric fields.

It is assumed that there exists some region in stress and electric field space within which the material responds in a linear elastic, piezoelectric and dielectric manner about the current state of remanent strain and remanent polarization. The surface bounding this region will be called the switching surface. The second law of thermodynamics and the postulate of maximum dissipation are satisfied if the switching surface is convex and encloses the origin in a modified stress and electric field space, and the increments of remanent strain and remanent polarization are normal to the switching surface during changes in remanency. The specific form of the switching surface used here is

$$
\Phi=\frac{3 \hat{s}_{i j} \hat{s}_{i j}}{2 \sigma_{0}^{2}}+\frac{\hat{E}_{i} \hat{E}_{i}}{E_{0}^{2}}+\frac{\beta\left(\hat{s}_{i j} \hat{E}_{i} P_{j}^{r}+\hat{s}_{i j} \hat{E}_{j} P_{i}^{r}\right)}{2 \sigma_{0} E_{0} P_{\max }}-1=0
$$

where the modified stress and electric field are

$$
\begin{aligned}
& \hat{\sigma}_{i j}=\sigma_{i j}-\frac{\partial \Psi^{r}}{\partial \varepsilon_{i j}^{r}} \quad \text { with } \hat{s}_{i j}=\hat{\sigma}_{i j}-\frac{\hat{\sigma}_{k k}}{3} \delta_{i j}, \text { and } \\
& \hat{E}_{i}=E_{i}-\frac{\partial \Psi^{r}}{\partial P_{i}^{r}}+\frac{\partial d_{j k l}}{\partial P_{i}^{r}} E_{j} \sigma_{k l}
\end{aligned}
$$

Then, during switching the remanent strain and remanent polarization increments are given by

$$
\begin{aligned}
\dot{\varepsilon}_{i j}^{r} & =\lambda \frac{\partial \Phi}{\partial \hat{\sigma}_{i j}} \\
& =\lambda\left[\frac{3 \hat{s}_{i j}}{\sigma_{0}^{2}}+\frac{\beta}{2 \sigma_{0} E_{0} P_{\max }}\left(\hat{E}_{i} P_{j}^{r}+\hat{E}_{j} P_{i}^{r}-\frac{2}{3} \hat{E}_{k} P_{k}^{r} \delta_{i j}\right)\right]
\end{aligned}
$$

and

$$
\dot{P}_{i}^{r}=\lambda \frac{\partial \Phi}{\partial \hat{E}_{i}}=\lambda\left[\frac{2 \hat{E}_{i}}{E_{0}^{2}}+\frac{\beta \hat{s}_{i j} P_{j}^{r}}{\sigma_{0} E_{0} P_{\max }}\right]
$$

Use of the consistency condition during switching, i.e. $\dot{\Phi}=0$, can be used to determine the switching multiplier $\lambda$ and the associated tangent moduli for the material. Details of these derivations are given in Landis (2002). Now, to have a final material specific form of the 
theory, the remanent potential $\Psi^{r}$ must also be specified. For the results to be presented in this section, $\Psi^{r}$ is split into a mechanical part $\Psi^{m}$ that enforces the strain saturation conditions and an electrical part $\Psi^{e}$ that enforces the polarization saturation conditions. The forms for each of these potentials are given as follows.

$$
\begin{gathered}
\Psi^{r}=\Psi^{m}+\Psi^{e} \\
\Psi^{m}=\frac{1}{2} H_{0}^{m} \varepsilon_{c}\left[\frac{J_{2}^{e}}{\varepsilon_{c}} \exp \left(\frac{m_{m}}{1-\bar{\varepsilon} / \varepsilon_{c}}\right)\right]^{2}
\end{gathered}
$$

where

$$
\bar{\varepsilon}=J_{2}^{e} f\left(J_{3}^{e} / J_{2}^{e}\right)
$$

and

$$
\begin{gathered}
f\left(\frac{J_{3}^{e}}{J_{2}^{e}}\right)=-0.0965\left(\frac{J_{3}^{e}}{J_{2}^{e}}\right)^{3}+0.01\left(\frac{J_{3}^{e}}{J_{2}^{e}}\right)^{6} \\
+0.8935, \quad \text { for }\left(\frac{J_{3}^{e}}{J_{2}^{e}}\right)<0 \\
f\left(\frac{J_{3}^{e}}{J_{2}^{e}}\right)=-0.1075\left(\frac{J_{3}^{e}}{J_{2}^{e}}\right)^{3}-0.027\left(\frac{J_{3}^{e}}{J_{2}^{e}}\right)^{6}-0.028\left(\frac{J_{3}^{e}}{J_{2}^{e}}\right)^{21} \\
+0.8935 \text { for }\left(\frac{J_{3}^{e}}{J_{2}^{e}}\right) \geq 0 .
\end{gathered}
$$

Then,

$$
\Psi^{e}=H_{0}^{e} P_{\max }\left[\ln \left(\frac{1}{1-P^{r} / P_{\mathrm{sat}}}\right)-\frac{P^{r}}{P_{\mathrm{sat}}}\right]
$$

where

$$
\begin{aligned}
& P_{\text {sat }}=\frac{P_{\max }-P_{\min }}{\varepsilon_{t}+\varepsilon_{c}}\left(\varepsilon_{i j}^{r} n_{i} n_{j}+\varepsilon_{c}\right)+P_{\min }, \quad \text { with } \\
& P_{\max }=0.83 P_{0} \text { and } P_{\min }=P_{\max } / 4
\end{aligned}
$$

The mechanical part of the remanent potential in Equation (4.11) enforces the remanent strain saturation conditions by forcing the potential to go to infinity as the newly defined remanent strain variable $\bar{\varepsilon}$ approaches the magnitude of the compressive strain saturation level $\varepsilon_{c}$. The primary result taken from the microelectromechanical simulations presented in Figure 3(b) is the functional fit $f\left(J_{3}^{e} / J_{2}^{e}\right)$ of Equations (4.13) and (4.14). This function is able to reproduce the numerical results of Figure 3(b) to within $0.15 \%$ as $\bar{\varepsilon}$ approaches $\varepsilon_{c}$.

Next, the electrical part of the potential given in Equation (4.15) enforces the polarization saturation conditions by causing the remanent potential to go to infinity as the magnitude of the remanent polarization $P^{r}$ approaches $P_{\text {sat }}$. Notice that $P_{\text {sat }}$ depends on the remanent strain state, and that the invariant $\varepsilon_{i j}^{r} n_{i} n_{j}$ has been used as a multiaxial generalization of the uniaxial results of Figure 4. Specifically, the multi-axial remanent polarization saturation limit is assumed to be a function of only $\varepsilon_{i j}^{r} n_{i} n_{j}$ implying that $\tilde{P}\left(J_{3}^{e} / J_{2}^{e}, J_{2}^{e} / \varepsilon_{0}, n_{1}, n_{2}\right)=$ $\tilde{P}_{\text {uni }}\left(\varepsilon_{i j}^{r} n_{i} n_{j} / \varepsilon_{0}\right)$. Recall that $\tilde{P}$ and $\tilde{P}_{\text {uni }}$ are described in Section 3.2. Furthermore, the accurate form of $\tilde{P}_{\text {uni }}\left(\varepsilon_{i j}^{r} n_{i} n_{j} / \varepsilon_{0}\right)$ is plotted on Figure 4 , and approximated as a straight line by Equation (4.16) for simplicity. The appropriateness of using the invariant $\varepsilon_{i j}^{r} n_{i} n_{j}$ within the polarization saturation potential as a generalization of the uniaxial remanent strain $\varepsilon^{r}$ to multiaxial remanent strain states can only be verified by more detailed microelectromechanical simulations. However, this generalization will not effect results generated for uniaxial situations where the applied stress and electric field are aligned along the same axis.

Finally, the $\beta$ term appearing in the switching surface has been included in the theory in order to achieve the best possible agreement with experimental observations. Extensive investigations have been carried out in search for functional forms of $\Phi$ with $\beta=0$. However, the results from these cases have not yet been able to reproduce the wide range of experimental observations presented in Lynch (1996) and Huber and Fleck (2001). It is possible that micro-electromechanical investigations may provide insight into these features of the model. However, such investigations will require the determination of the driving forces for changes in remanency and the assumption of isotropic linear properties for the single crystals as in Equations (2.4) and (2.5) will not be valid.

Figure 5(a)-(e) illustrate the uniaxial predictions of the micro-electromechanically informed phenomenological model presented in this section for (a) the electric displacement versus electric field hysteresis loop, (b) the strain versus electric field butterfly loop, (c) the electric displacement versus stress during mechanical depolarization, (d) the strain versus stress during mechanical depolarization and (e) the ferroelastic stress versus strain hysteresis. Overall, the qualitative agreement of these predicted loops with the experimental observations of Lynch (1996) is excellent. From these curves there are a few observations worth noting. On Figures 5(a) and (b), reverse switching due to Bauschinger effects is occurring when $E=0$ after the reversal of the electric field. This implies that the slopes of the hysteresis and butterfly loops at $E=0$ are not indicative of the linear dielectric and piezoelectric coefficients of the material. Instead, the slopes of these loops just after the reversal of the electric field are the best measures of these linear properties. A similar argument can be made for the piezoelectric and elastic properties at the beginning of the mechanical depolarization in Figures 5(c) and 
(a)

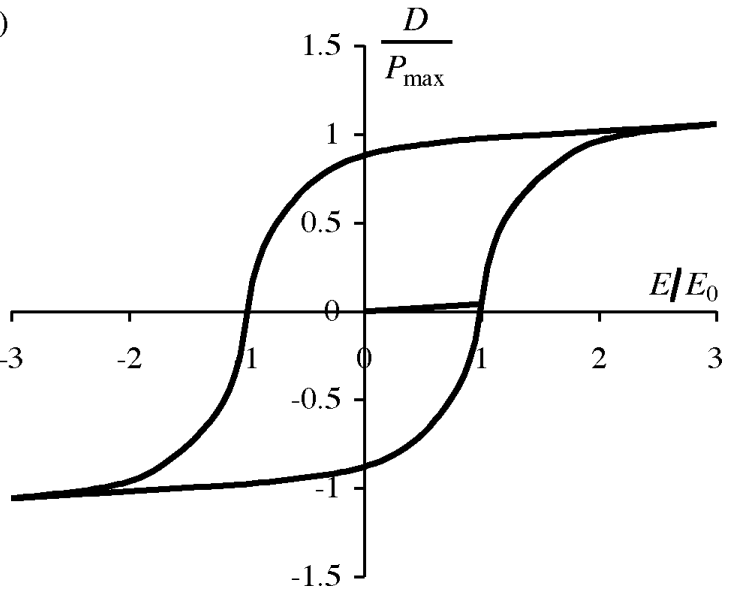

(c)

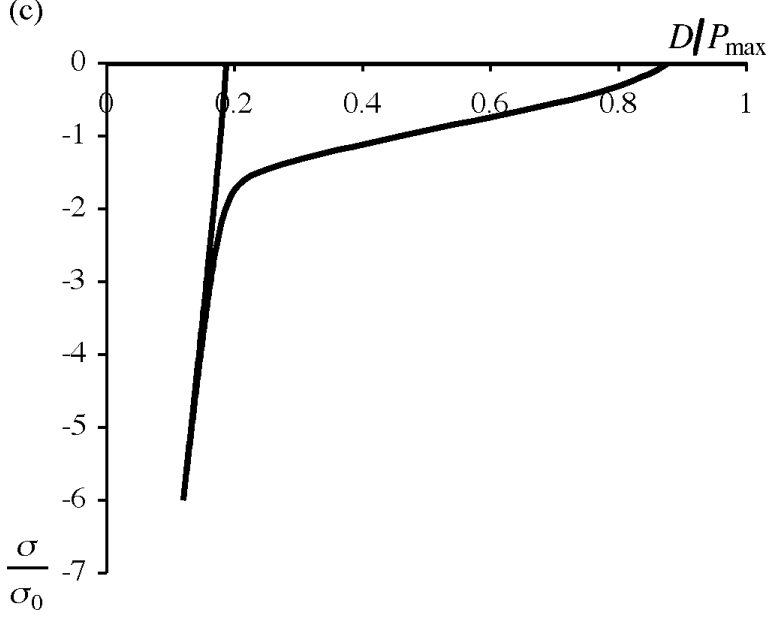

(b)

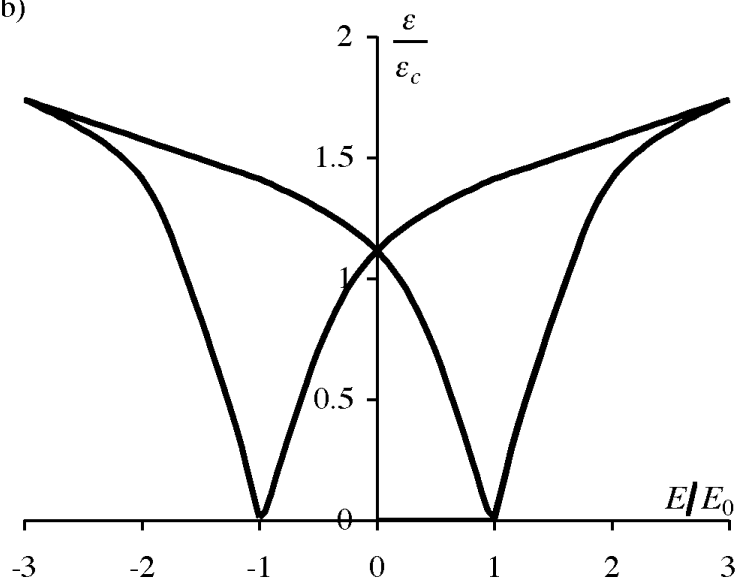

(d) $\varepsilon / \varepsilon_{c}$

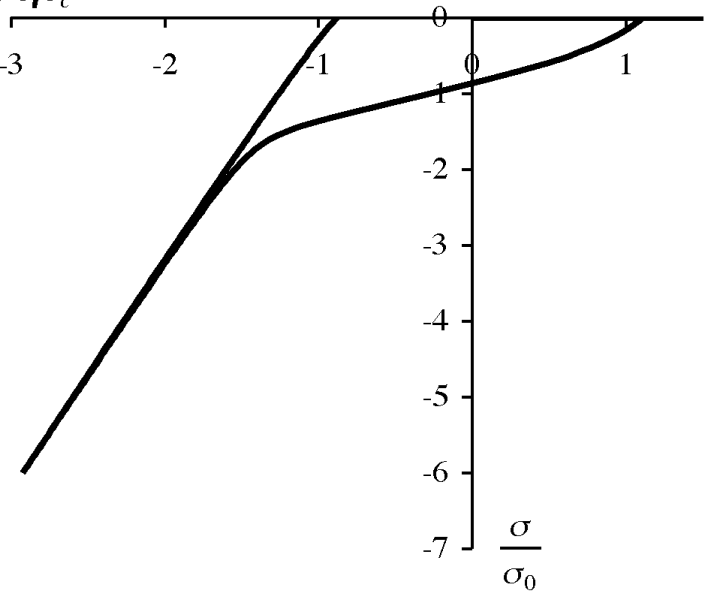

(e)

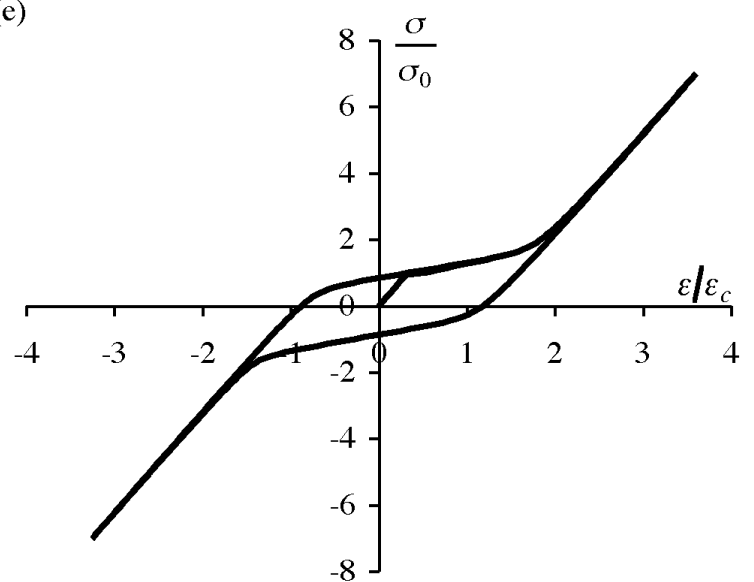

Figure 5. (a) The electric displacement vs. electric field hysteresis loop; (b) The strain vs. electric field butterfly loop; (c) The electric displacement vs. stress during mechanical depolarization; (d) The strain vs. stress during mechanical depolarization; (e) The ferroelastic stress vs. strain hysteresis loop. All curves represent uniaxial electromechanically coupled behavior. The model parameters used to generate these curves are $\sigma_{0}=27.5 \mathrm{MPa}, E_{0}=0.35 \mathrm{MV} / \mathrm{m}, P_{\max }=0.26 \mathrm{C} / \mathrm{m}^{2}, \varepsilon_{c}=0.12 \%, \quad \beta=2.95, \kappa=3 \times 10^{-8} \mathrm{C} /(\mathrm{mV}), \mu=25 \mathrm{GPa}, \nu=0.4$, $d_{33}=6 \times 10^{-10} \mathrm{~m} / \mathrm{V}, m_{m}=0.01, H_{0}^{m}=0.5 \sigma_{0}$ and $H_{0}^{e}=0.05 E_{0}$.

(d). The Young's modulus of the material should be taken from the unloading portion of the stress versus strain during depolarization curve, and not at the initial application of stress. Also, notice the asym- metry in the achievable tensile and compressive strains during purely ferroelastic stress-strain response, i.e. larger strain magnitudes occur in tension than in compression. 

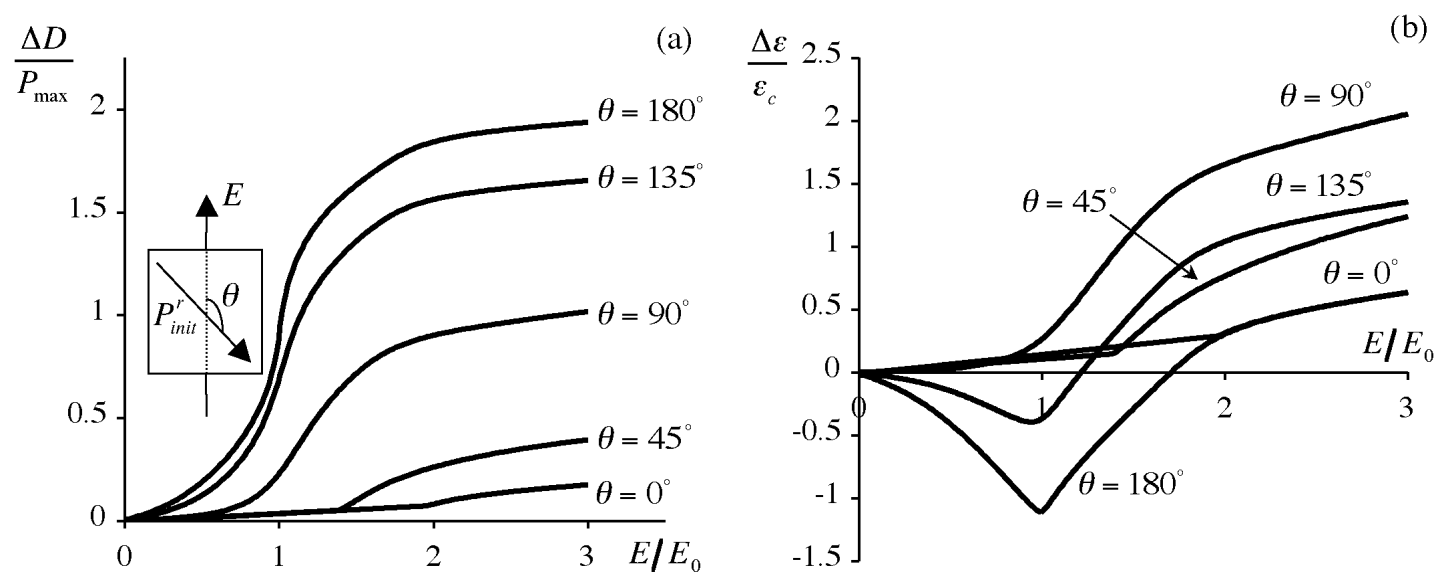

Figure 6. Simulation of the polarization rotation experiments of Huber and Fleck (2001). Electric field, E, is applied at an angle $\theta$ to an initial remanent polarization direction. The (a) electric displacement change $\Delta D$ and the (b) axial strain change $\Delta \varepsilon$ in the direction of the applied electric field are plotted vs. the electric field for a range of $\theta$ values.

Finally, the multi-axial polarization rotation experiments of Huber and Fleck (2001) are simulated within the theory and plotted on Figures 6(a) and (b). The experiment is performed as follows. Initially, an unpoled specimen is poled by a strong electric field. Then, a new specimen is cut and coated with electrode material such that the initial polarization direction is at an angle of $\theta$ to the new electric field direction which is perpendicular to the electrodes. An electric field of magnitude $E$ is applied to the specimen and the electric displacement change $\Delta D$ and axial strain change $\Delta \varepsilon$ in the direction of the applied electric field are measured. In the original experiments by Huber and Fleck only the electric displacement changes were measured. The qualitative agreement between the simulations displayed on Figure 6(a) and measurements of Huber and Fleck is exceptional. The corresponding strain changes plotted in Figure 6(b) are included as possible predictions for future experimental observations of strain for this type of experiment.

\section{DISCUSSION}

In this paper, micro-electromechanical simulations have been carried out to determine the possible states of remanent strain and remanent polarization in ferroelectric polycrystals composed of single crystals with tetragonal crystal structure. These simulations were used to map out the entire multi-axial range of remanent strain states as well as the uniaxial polarization states. It was quantitatively shown how the remanent polarization saturation limit depends on the level of remanent strain in the material. The saturation information obtained from the micro-electromechanical simulations was then fed into a phenomenological framework in order to ensure that the phenomenological model could not produce unattainable combinations of remanent strain and remanent polarization. The predictions of the phenomenological model are found to be in excellent qualitative accord with experimental observations of polycrystalline material behavior.

The results generated with the micro-electromechanical model are specific to untextured polycrystals with tetragonal crystal structure. However, similar simulations could be carried out for different crystal structures, e.g. orthorhombic or rhombohedral, or for textured polycrystals. Different crystal structures would effect the descriptions of the variant strains and polarizations of Equations (2.2) and (2.3), and polycrystalline texture would change the self-consistent averaging technique by altering the volume concentrations of the different orientations of the single crystals. In either of these situations, or a combination of the two, micro-electromechanical simulations can be used to map out the remanent strain and polarization states that are possible in a given ferroelectric polycrystalline material.

\section{ACKNOWLEDGMENT}

The authors would like to acknowledge support from the Army Research Office contract DAAD19-02-1-0241 for JW and CML, and from the Office of Naval Research contract N00014-03-1-0537 for JS and CML.

\section{REFERENCES}

Cocks, A.C.F. and McMeeking, R.M. 1999. "A Phenomenological Constitutive Law for the Behavior of Ferroelectric Ceramics," Ferroelectrics, 228:219-228.

Frölich, A., 2001, "Mikromechanisches Modell zur Ermittlung Effektiver Materialeigenschaften von Piezoelektrischen Polykristallen," Dissertation Thesis, Universität Karlsruhe, Germany. 
Hill, R. 1965. "Continuum Micro-mechanics of Elastoplastic Polycrystals," Journal of the Mechanics and Physics of Solids, 13:89-101.

Hill, R. 1967. "The Essential Structure of Constitutive Laws for Metal Composites and Polycrystals," Journal of the Mechanics and Physics of Solids, 15:79-95.

Huber, J.E., Fleck, N.A., Landis, C.M. and McMeeking, R.M. 1999. "A Constitutive Model for Ferroelectric Polycrystals," Journal of the Mechanics and Physics of Solids, 47:1663-1697.

Huber, J.E. and Fleck, N.A. 2001. "Multi-axial Electrical Switching of a Ferroelectric: Theory versus Experiment," Journal of the Mechanics and Physics of Solids, 49:785-811.

Hutchinson, J.W. 1970. "Elastic-plastic Behavior of Polycrystalline Metals and Composites," Proceedings of the Royal Society of London, A319:247-272.

Hwang, S.C. and McMeeking, R.M. 1998. "A Finite Element Model of Ferroelectric Polycrystals," Ferroelectrics, 211:177194.

Hwang, S.C. and McMeeking, R.M. 1999. "A Finite Element Model of Ferroelastic Polycrystals," International Journal of Solids and Structures, 36:1541-1546.
Hwang, S.C., Huber, J.E., McMeeking, R.M. and Fleck, N.A. 1998. "The Simulation of Switching in Polycrystalline Ferroelectric Ceramics," Journal of Applied Physics, 84:1530-1540.

Hwang, S.C., Lynch, C.S. and McMeeking, R.M. 1995. "Ferroelectric/ Ferroelastic Interactions and a Polarization Switching Model," Acta Materialia, 43:2073-2084.

Kamlah, M. 2001. "Ferroelectric and Ferroelastic Piezoceramics Modeling and Electromechanical Hysteresis Phenomena," Continuum Mechanics and Thermodynamics, 13:219-268.

Landis, C.M. 2002. "Fully Coupled, Multi-axial, Symmetric Constitutive Laws for Polycrystalline Ferroelectric Ceramics," Journal of the Mechanics and Physics of Solids, 50:127-152.

Landis, C.M. 2003. "On the Strain Saturation Conditions in Polycrystalline Ferroelastic Materials," Journal of Applied Mechanics, 70:470-478.

Lu, W., Fang, D.N. Li, C.Q. and Hwang, K.C. 1999. "Nonlinear Electric-mechanical Behavior and Micromechanics Modelling of Ferroelectric Domain Evolution," Acta Materialia, 47:2913-2926.

Lynch, C.S. 1996. "The Effect of Uniaxial Stress on the Electromechanical Response of 8/65/35 PLZT," Acta Materialia, 44:41374148 . 